\title{
Conflicto Interparental y Acoso Escolar: Evaluación del Rol Mediador de la Mentalización y Regulación Emocional
}

\author{
Interparental Conflict and Bullying: Assessment of the Mediating Role of \\ Mentalization and Emotion Regulation
}

\author{
Marina Ferreras Picazo $^{1}$, Susana Cormenzana Redondo ${ }^{2}$ y Ana Martínez-Pampliega ${ }^{3}$
}

\begin{abstract}
Resumen
El presente estudio tiene como objetivo profundizar en el papel mediador de la mentalización y la regulación emocional en la relación entre el conflicto parental y el acoso escolar. En el estudio participaron 385 estudiantes españoles de entre 12 y 17 años $(M=13.94 ; D . T .=1.80)$. Los resultados obtenidos a través de Process permitieron confirmar cómo la percepción del conflicto interparental se relaciona con el acoso escolar de manera indirecta a través de una mediación múltiple entre las variables hipomentalización y desregulación emocional, siendo el efecto mediador de esta última más notable. Este resultado fue constatado fundamentalmente en relación a conductas de victimización, así como para la puntuación total del instrumento de acoso. Los resultados permiten destacar el papel preventivo del abordaje de la mentalización y regulación emocional en la incidencia de acoso escolar en contexto de conflicto interparental.
\end{abstract}

Palavras-chave: acoso escolar, conflicto interparental, hipomentalización, regulación emocional, mentalización

\begin{abstract}
The aim of the present study is to delve into the mediating role of the mentalization and emotion regulation ability in relation to interparental conflict and bullying. The sample was comprised of 385 Spanish students aged between 12 and 17 years old $(M=13.94 ; D . T .=1.80)$. The results obtained through Process made it possible to confirm how interparental conflict is indirectly associated with bullying through multiple mediation among the hypomentalization and emotion dysregulation variables, while the mediating effect of the latter was more notable. These results were mainly found with regard to victimization behaviors as well as for the total score of the bullying instrument. These results highlight the preventive role that the approach of mentalization and emotion regulation may have in the incidence of bullying in the interparental conflict context.
\end{abstract}

Keywords: bullying, interparental conflict, hipomentalization, emotion regulation, mentalization

\footnotetext{
${ }^{1}$ Psicóloga. NOÛS Instituto de Psicoterapia Integrativa. Facultad de Ciencias de la Salud. Departamento de Psicología, Universidad de Deusto. Avda. Universidades 24, 48007 Bilbao (Bizkaia), España. Correo: marina.ferreras.picazo@ gmail.com (Autor de correspondencia)

${ }^{2}$ Doctora en Psicología. Facultad de Ciencias de la Salud. Departamento de Psicología. Docente e Investigadora del grupo Deusto Familypsych, Universidad de Deusto, Avda. Universidades 24, 48007 Bilbao (Bizkaia), España. Tel.: +34-944139000 ext-2756. Correo: susana.cormenzana@ deusto.es

${ }^{3}$ Catedrática de Psicología. Facultad de Ciencias de la Salud. Departamento de Psicología. Equipo de investigación Deusto FamilyPsych. Universidad de Deusto, Avda. Universidades 24, 48007 Bilbao (Bizkaia), España. Tel.: +34-944139174. Correo: martinez.pampliega@ deusto.es 


\section{Introducción}

El acoso escolar es un fenómeno global, el cual presenta consecuencias negativas tanto para víctimas como para acosadores (Due et al., 2005; Salmivalli; 2010; UNESCO, 2018). Según un informe realizado por la UNESCO (2018), mientras en algunos países del mundo se ha observado una reducción en la presencia de este fenómeno en los últimos años, en otros no se han experimentado cambio o incluso se ha producido un aumento de su prevalencia. Según este informe, uno de cada tres estudiantes (32\%), señaló haber recibido conductas de acoso durante el último mes por sus compañeros/as en su centro escolar.

El bullying o acoso escolar es definido como el ataque o intimidación físico, psicológico y/o verbal dirigido a causar daño o angustia a una víctima concreta (Christie-Mizell, 2003). Este incluye la intencionalidad de la agresión, un desequilibrio de poder entre el agresor y la víctima, la cual no puede defenderse fácilmente por sí misma, y la repetición de la acción en el tiempo (Save the Children, 2013). Las manifestaciones más comunes de este tipo de conducta de acoso son los insultos directos o indirectos, rumores, robo de pertenencias, amenazas o golpes (Save the Children, 2016, Unnever, 2005).

Las formas de acoso escolar ocurren a lo largo de un continuo en el que se contemplan diferentes roles, que van desde agresor, víctima y agresorvíctima (Holt, Finkelhor, \& Kaufman-Kantor, 2007; Unnever, 2005). Los/as menores denominados agresores son aquellos perpetradores de la conducta de acoso, que no experimentan una victimización significativa por los/as compañeros. Las víctimas son objeto de la conducta de acoso por parte de los/as compañeros/as sin ser perpetradores de conducta agresiva. Finalmente, los/as agresores-víctimas son aquellos niños/as que desempeñan simultáneamente ambos roles, el de objetivo y perpetrador de conducta de acoso, pudiendo reaccionar de forma agresiva como defensa ante el rol de víctima (Holt et al., 2007).

\section{Relación entre el Acoso Escolar y Conflicto Interparental}

La relación entre el desarrollo del acoso escolar y el conflicto interparental destructivo ha sido destacada en diversos estudios (ej, ChristieMizell, 2003; Martínez, Musitu, Amador, \& Murgui, 2009; Mistli, 2017), señalando esta relación para los diversos roles dentro del acoso escolar (Ahmed \& Braithwaite, 2004; Martínez et al., 2009; Morris, 2007). El conflicto interparental destructivo se caracteriza por conductas violentas, ataques agresivos verbales o físicos y la retirada o evitación del conflicto, que lleva a dejarlo sin resolver, mostrando poca capacidad resolutiva ante el conflicto entre los padres (Cummings \& Davies, 2010; Delgado, Romero, Antolín-Suárez, \& Parra, 2016). Esta ausencia en el uso de tácticas de resolución de conflicto apropiadas, influye en los menores a la hora de adquirir estrategias adecuadas para resolver conflictos. Diversos estudios muestran que el uso de estas estrategias dentro del grupo de iguales aumenta el riesgo de victimización (Goeke-Morey, Cummings, \& Papp, 2007; Schwartz, Petit, Dodge, \& Bates, 2000). En relación al rol agresivo, la exposición a situaciones de conflicto interparental se relaciona con una mayor predisposición en los hijos/as a la hostilidad, conducta antisocial y violencia escolar, presentando una mayor aceptación de comportamientos agresivos como medio para lograr sus objetivos (Buelga, Iranzo, Cava, \& Torralba, 2015; Ingoldsby, Shaw, \& García, 2001). Así, la investigación pone de manifiesto que ser testigo de este tipo de interacciones favorece la integración de modelos no adaptativos de resolución de conflicto lo cual aumenta el riesgo en los menores de victimización y agresión entre iguales.

Uno de los modelos de referencia en la explicación de la vinculación entre el conflicto interparental y las respuestas desadaptativas, como el acoso escolar, es la hipótesis de seguridad emocional (Davies \& Cummings, 1994). Según esta hipótesis, la posible reducción de disponibilidad emocional o sensibilidad de los padres, así como el estrés generado en los hijos/as ante la vivencia del conflicto parental (Davies \& Cummings, 1994; Gong, 2013; Krishnakumar \& Buehler, 2000; Warmuth, Cummings, \& Davies, 2018), afecta a la seguridad emocional, la cual a su vez tendrá un impacto en el funcionamiento del niño/a a través de varios procesos interrelacionados. En primer lugar, cuando el clima emocional de un niño/a es negativo, 
coercitivo o impredecible, este se encuentra en riesgo de desarrollar problemas de regulación emocional debido a las frecuentes exposiciones a situaciones de conflicto, así como a la posible presencia de manipulaciones emocionales (Davies \& Martin, 2013; Morris, 2000). La presencia de niveles altos de conflicto y la dificultad derivada en la capacidad de regulación emocional, tiene un impacto en el tipo de representaciones cognitivas que los/as menores desarrollan, lo que conduce a conductas de afrontamiento menos adaptativas y más desreguladas con el fin de poder reducir o finalizar el conflicto parental y así aumentar su sensación de seguridad emocional (Davies \& Cummings, 1994; Cummings, George, McCoy, \& Davies, 2012). Esta hipótesis ofrece un mayor entendimiento a la influencia que la presencia de conflicto interparental tiene en el riesgo de los menores de ubicarse en un rol de agresión o victimización en situación de acoso escolar a través de una adquisición y uso de tácticas de resolución de conflicto poco adaptativas.

\section{Regulación Emocional y su relación con el Conflicto Parental y el Acoso Escolar}

Desde la hipótesis de seguridad emocional, la regulación emocional es una variable potencial para comprender el desarrollo de conductas desadaptativas en niños/as y adolescentes en situación de conflicto interparental destructivo. La regulación emocional ha sido definida como el proceso extrínseco e intrínseco responsable de monitorizar, evaluar y modificar las reacciones emocionales, siendo su objetivo la adaptación del individuo (Gong, 2013; Thompson, 1994). Por el contrario, se identifica la desregulación emocional como la dificultad de responder a los estímulos con control emocional (Keenan, 2000).

Como respuesta al conflicto, el niño/a puede sentir tristeza, miedo, alivio o felicidad, dependiendo de cómo el conflicto entre los padres sea expresado y manejado. Cuando un cuidador/a ignora estas emociones a menudo, como ocurre en situación de reducción de disponibilidad parental, el niño/a aprenderá a minimizar o maximizar la expresión de esta emoción para adaptarse al estilo de respuesta del cuidador/a (Davies \& Cummings, 1994). Las implicaciones de este funcionamiento en el niño se ven determinadas por cuánto el niño/a siente estas emociones y cómo este puede regularlas de manera adaptativa (Harold, Shelton, Goeke-Morey, \& Cummings, 2004).

Diversos estudios confirman la relación entre el conflicto parental, el acoso escolar y la regulación emocional, mostrando como el conflicto parental destructivo se asocia con problemas de regulación emocional en niños/as así como con dificultades en manejar emociones negativas como la rabia o la tristeza (Gong, 2013; Koss et al., 2011). Esta inestabilidad emocional se presenta como un fuerte predictor en la conducta agresiva (Estévez, Carillo, \& Gómez, 2018; TurPorcar, Mestre, Samper, \& Malonda, 2012), como es el caso de los acosadores, quienes señalan tener un mayor enfado y mayor impulsividad (Espelage \& Swearer, 2003). En relación al rol de victimización, los menores que tienden a responder con respuestas emocionales inapropiadas e inflexibles a situaciones sociales, se encuentran en un mayor riesgo de rechazo por parte de sus compañeros (Estévez et al., 2018; Kelly, Schwart, Gorman, \& Nakamoto, 2008; Pope \& Bierman, 1999).

\section{Mentalización y su relación con la Regulación Emocional, Conflicto Interparental y Acoso Escolar}

Un concepto estrechamente vinculado a la regulación emocional es el de mentalización (Allen, 2008), una capacidad dinámica afectada por el estrés y la activación, particularmente en el contexto de las relaciones de apego (Bleiberg 2013). La mentalización ha sido definida como el intento, consciente o inconsciente, de comprender los estados mentales (entre los que se encuentran deseos, emociones, creencias) en uno mismo y en los demás, así como la capacidad de interpretar y predecir de manera precisa, pero provisional, las conductas e interacciones interpersonales motivadas por estos estados mentales subyacentes (Allen, 2008; Fonagy, 1991; Fonagy, Steele, \& Steele, 1991). Como resultado de la mentalización, el individuo reacciona al comportamiento que observa de otra persona (Bateman \& Fonagy, 2012).

Se observan dos dificultades en la capacidad de mentalización. La primera, denominada, hipomentalización, refleja la dificultad de considerar modelos complejos en los estados mentales en la propia mente y/o en la de los 
demás, lo que equivale a una limitación en el entendimiento de uno mismo y/o otros, reflejando incertidumbre ante estos estados mentales. La tendencia opuesta, denominada hipermentalización, es la generación de representaciones de los estados mentales de uno mismo y/o otro sin la apropiada evidencia disponible para apoyar esos modelos, mostrando una excesiva certeza en a la hora de considerar estos estados mentales (Fonagy, et al., 2016).

En el modelo de Fonagy y Target (1997), ambos autores plantean que una adecuada mentalización permite a los cuidadores contener y marcar las necesidades emocionales del niño/a a través de sus reacciones, mostrando que esos sentimientos desagradables pueden ser regulados, siendo la influencia de la mentalización fundamental en la regulación emocional, ya que ante una dificultad en la habilidad de reconocer e interpretar los estados mentales, la regulación emocional puede ser insuficiente o inapropiada (Allen, 2008). Esta mentalización del padre hacia el niño/a puede verse limitada por la reducción de disponibilidad emocional por parte de los padres, debido a momentos de altos niveles de estrés o ansiedad como ocurre en caso de conflicto interparental (Davies \& Cummings, 1994; LyonsRuth, Bronfman, \& Atwood, 1999; Werneck, Eder, Yanagida, \& Rollett, 2014).

En relación al acoso escolar la mentalización ha sido estudiada fundamentalmente en relación a la conducta agresiva, y rara vez en relación al desarrollo del acoso en contexto escolar, así como en situación de victimización. En esta línea, en relación al rol agresor, la literatura señala que la dificultad en la capacidad de mentalización puede influir en la capacidad de regular la impulsividad y de forma relacionada, inhibir los comportamientos violentos (Bateman, Bolton, \& Fonagy, 2013, Fonagy \& Luyten, 2009). Sin embargo, los mecanismos a través del cual la mentalización regulan estas respuestas, aún están por explorar (Morosan, Fonseca-Pedrero, \& Debanné, 2020). Respecto a las víctimas, la dificultad en su capacidad de mentalización se relaciona con su habilidad para observar y controlar sus sentimientos, evaluar señales del acosador e integrar esa información intra e interpersonal lo que influye en su capacidad para desarrollar una estrategia de enfrentamiento efectiva (Mahady-Wilton, Craig, \& Pepler, 2000), perpetuando su rol de víctima en situación de acoso escolar.

Las investigaciones relativas al estudio de la capacidad mentalizadora y la regulación emocional se han focalizado principalmente en niños/as y adultos/as, siendo escasas las referencias con población adolescente. No obstante, los escasos estudios existentes ponen de relieve la importancia del periodo adolescente para el desarrollo de la capacidad de mentalización y su asociación con la regulación emocional (Benbassat \& Priel, 2015; Marszał \& Jańczak, 2017), siendo la etapa adolescente un periodo de transición, crucial para el desarrollo de procesos psicológicos como la habilidad de vincular estados mentales con comportamientos, y de forma relacionada, con la capacidad de regulación emocional (Allen, 2008; Blakemore, 2008).

\section{Objetivo e hipótesis}

La revisión de la literatura señala la relación, directa e indirecta, existente entre, por un lado, el conflicto interparental y el acoso escolar para los diferentes roles descritos (víctima y acosador) y por el otro, las habilidades de regulación emocional y la capacidad de mentalización. Sin embargo, no existen estudios dirigidos a comprender el proceso a través del cual la regulación emocional y la mentalización podrían contribuir a esclarecer la relación entre el conflicto interparental y el acoso escolar. Por ello, el presente estudio se dirige a comprobar este papel mediador de las variables indicadas, regulación emocional y la mentalización, en el acoso escolar entre adolescentes expuestos a conflicto interparental. Se parte de la hipótesis de que en situaciones de conflicto interparental, los hijos/as mostrarán una mayor desregulación emocional y una menor mentalización, lo cual, a su vez, se relacionará con el acoso escolar, tanto para aquellos menores en rol de agresor como para el rol de victimización, así como que las variables de desregulación emocional y mentalización mediarán la relación entre percepción de conflicto parental y acoso escolar para ambos roles. 


\section{Método}

\section{Participantes}

En el estudio formaron parte 385 estudiantes que se encontraban cursando Enseñanza Secundaria Obligatoria (ESO) en dos municipios de Bizkaia, cuyas edades comprendían entre los 12 y 17 años $(M=13.94 ; D . T .=1.80)$. Respecto al sexo, de aquellos participantes que completaron todos los datos, un 53.64\% ( $n=206)$ fueron chicos y un $46,26 \%(n=178)$ fueron chicas.

\section{Instrumentos}

Cuestionario del Proyecto Europeo de Intervención en Acoso Escolar (European Bullying Intervention Project Questionnaire, EBIPQ; Brighi et al., 2012), adaptado al castellano por Ortega-Ruiz, Del Rey y Casas (2016). El autoinforme está compuesto por 14 ítems tipo Likert con cinco opciones de respuesta $(0=$ No, $1=$ Sí, una o dos veces, $2=$ Sí, una o dos veces al mes, $3=$ Sí, alrededor de una vez a la semana y $4=$ Sí, más de una vez a la semana), con una mayor puntuación indicando una mayor ocurrencia. Presenta dos subescalas, la primera formada por los siete primeros ítems la cual evalúa aspectos relacionados con la victimización y una segunda escala en correspondencia con la agresión, así como la puntuación total que engloba las conductas en su conjunto. Todos comprenden ítems idénticos, cambiando el punto de vista según víctima o agresor. Los ítems evalúan comportamientos como abuso físico directo (ej.: "Alguien me ha golpeado, me ha pateado o me ha empujado"), abuso indirecto (ej. "Alguien ha difundido rumores sobre mí"), abuso verbal (ej.: "He dicho a otras personas palabras malsonantes sobre alguien"), abuso psicológico (ej.: "He amenazado a alguien") y exclusión social (ej.: "He excluido o ignorado a alguien"). El tiempo de referencia para evaluar la frecuencia de implicación ha sido de los últimos dos meses. Los índices de consistencia interna alfa de Cronbach y omega de McDonald han mostrado ser adecuados (para victimización $\alpha=.80, \omega=.85$; para agresión $\alpha=.79, \omega=.81$ y para el total $\alpha=.84$ y $\omega=.85$ ).

Escala de percepción de los hijos/as del Conflicto interparental (Children's Perception of Interparental Conflict Scale, CPIC; Grych, Seid y Finchman, 1992), adaptada al castellano por
Iraurgi, Martínez-Pampliega, Sanz, Cosgaya, Galíndez y Muñoz (2006, 2008). Se ha utilizado la versión breve de 36 ítems distribuidos en tres grandes dimensiones y nueve subescalas: 1) Propiedades del conflicto, que integra las subescalas de Intensidad, Frecuencia, Estabilidad y Resolución (ej.: "Mis padres hacen las paces después de discutir"), 2) Vivencia o evaluación amenazante, que integra las subescalas de Eficacia de afrontamiento, Amenaza percibida, Triangulación (ej.: "Cuando mis padres discuten, temo que algo malo me ocurra") y 3) Culpabilidad con las subescalas de Contenido y Autoculpa (ej.: "Yo soy el culpable cuando mis padres discuten"), que recoge también la puntuación total. Los ítems presentan tres opciones de respuesta ( $1=$ Verdadero, $2=$ Casi verdadero y $3=$ Falso). La versión abreviada en muestra española ha mostrado una alta consistencia interna, con un alpha de Cronbach de .91. Las diferentes subescalas por separado presentan una consistencia interna de moderada a satisfactoria, oscilando desde .62 a .82 (Iraurgi et al., 2008).

Escala de dificultades en la regulación emocional (Difficulties in Emotion Regulation Scale, DERS; Gratz \& Roemer, 2004), adaptado para adolescentes por Gómez-Simón, Penelo y de la Osa (2014). La DERS está formada por 36 ítems que evalúa varios aspectos de la desregulación emocional a través de seis factores: Conciencia (ej.: "Estoy atento a mis emociones"), Descontrol (ej.: "Cuando me siento molesto, me cuesta controlar mi comportamiento"), Rechazo emocional (ej.: "Cuando estoy molesto, me enfado conmigo mismo por sentirme de esa manera"), Interferencia (ej.: "Cuando estoy molesto, me cuesta centrarme en las cosas"), Claridad (ej.: "Sé reconocer mis emociones") y Estrategias (ej.: "Tardo mucho tiempo en sentirme mejor"). El formato de respuesta es tipo Likert con cinco opciones de respuesta (siendo 1=Casi nunca a $5=$ Casi siempre), con una mayor puntuación indicando mayor dificultad en regulación emocional. Para Gómez-Simón, Panelo y de la Osa (2014) muestra una consistencia interna satisfactoria $(\alpha=.88)$. Las diferentes escalas por separado presentan una consistencia interna de moderada a satisfactoria (desde $\alpha=.62$ a .84).

Cuestionario de Funcionamiento Reflexivo (The Reflective Functioning Questionnaire 8, 
RFQ; Fonagy et al. (2016) (versión traducida al castellano proporcionada por los propios autores). El cuestionario consta de 8 ítems, dividido en dos subescalas: Certeza sobre los estados mentales (RFQ_C) e Incertidumbre sobre los estados mentales (RFQ_U). La primera se centra en la medida en que las personas no están de acuerdo con declaraciones como "siempre sé lo que siento" reflejando la hipermentalización. La subescala Incertidumbre sobre los estados mentales, refleja la hipomentalización. Respuestas tales como "los pensamientos de la gente son un misterio para mi" reflejan dicha parte. Las respuestas son de tipo Likert con 7 opciones de respuesta (1=Muy en desacuerdo a 7=Muy de acuerdo), con una mayor puntuación indicando una mayor dificultad en mentalización, ya sea tendiente a la certeza o incertidumbre según la escala. Las diferentes subescalas muestran diversos valores en la consistencia interna, siendo el alfa de Cronbach para la escala de Certeza en la muestra clínica .77 y para la escala Incertidumbre .65. En la muestra no clínica, la escala de Certeza obtuvo una fiabilidad de $.63 \mathrm{y}$ para la de Incertidumbre .67 (Fonagy et al., 2016). Se ha aplicado la versión breve para evitar efectos como la fatiga y por la recomendación de los autores de la versión breve para la investigación.

\section{Procedimiento}

La investigación fue trasversal, llevando a cabo la recogida de datos en dos centros educativos de Bizkaia durante el curso escolar 2017-2018. En cada uno de ellos se solicitó el permiso a la dirección para aplicar los cuestionarios. Una vez obtenido, se consultó a los docentes y estos entregaron a los alumnos el documento de consentimiento informado parental. $\mathrm{La}$ participación fue voluntaria y únicamente aquellos que mostraron autorización paterna o materna completaron los cuestionarios. Los cuestionarios fueron respondidos en su propia aula, de forma anónima y en presencia física del equipo de campo, quienes realizaron la presentación inicial de la tarea y estuvieron disponibles para solucionar las posibles dudas existentes. La respuesta a los cuestionarios tuvo lugar durante el horario de tutorías, con una duración promedio de 50 minutos. La pasación contó con la aprobación del comité ético de la universidad.

\section{Análisis estadísticos}

En primer lugar, se recodificaron las puntuaciones en aquellos ítems inversos, con una mayor puntuación indicando mayor presencia del constructo. Debido a la existencia de valores perdidos en algunos de los tests, se remplazaron mediante el método de mediana de puntos adyacentes, siempre y cuando el número de valores perdidos no excediera del $10 \%$ en una escala o subescala. Para la descripción de las características de los participantes y los índices de los instrumentos empleados, se utilizaron procedimientos de recuento de las frecuencias y sus porcentajes en el caso de variables cualitativas y estadísticos de tendencia central (Media - $M$ ) y dispersión (Desviación típica - DT) para las variables cuantitativas. Para el conjunto de instrumentos y subescalas utilizados se analizó la fiabilidad a través del coeficiente alpha de Cronbach $(\alpha)$ y omega de McDonald $(\omega)$, este último, siguiendo las recomendaciones de Trizano-Hermosilla y Alvarado (2016). Posteriormente, y debido a que no se cumplieron las pruebas de normalidad, se procedió a analizar la correlación a través de Spearman $(\rho)$, siguiendo para su interpretación el criterio de Cohen (1988) para ciencias de la conducta, que establece que un valor de $\rho$ por debajo .10 es una correlación pequeña, .30 es media y elevada a partir de .50 . Tras analizar las asociaciones lineales entre las variables de estudio y comprobar la correlación significativa entre ellas se procedió a comprobar las hipótesis de mediación múltiple. Para ello, se llevaron a cabo los análisis mediante las macros ofrecidas por Hayes y Preacher (2013) para su implementación en el SPSS. Posteriormente se compararon los efectos indirectos en los modelos de mediación significativos. Mediante el boostrap, se ofrece la estimación para el valor de los efectos en el modelo de mediación múltiple: si el intervalo de confianza incluye el valor cero (0), el efecto se interpreta como estadísticamente no significativo. Para interpretar los resultados de análisis de mediación se ha elegido un nivel de significación de $p<.05$ para un intervalo de confianza del 95\% (Boostraping=10.000, modelo 6). Los análisis indicados se realizaron con los programas SPSS para Windows en su versión 20.0, Jamovi en su versión 1.6.23 y Process en su versión v3.0. 


\section{Resultados}

En la Tabla 1 se muestran los descriptivos, la fiabilidad y la prueba Kolmogorov Smirnov para los instrumentos y subescalas utilizados. Los instrumentos y sus subescalas muestran niveles de consistencia interna adecuados, por encima de un valor de .70 tanto para el alpha de Cronbach como para la omega de McDonald. Las medias obtenidas muestran heterogeneidades por las diferencias en la medición en las escalas. Así, la media presentada de la puntuación total del EBIPQ y las subescalas del EBIPQ y el RFQ tienden a ser bajas, dentro de un rango de 0 a 30 para el EBIPQ y de 0 a 3 para el RFQ. Para el total de las escalas CPIC y DERS la media obtenida tiende a valores medios, difiriendo de los valores obtenidos para las subescalas, con valores que tienden a ser de medio-bajos. A continuación, en la Tabla 2 se presentan las matrices de correlación entre las puntuaciones globales y subescalas de los instrumentos utilizados.

De acuerdo con los resultados, la subescala agresión se correlacionó significativamente, y de forma elevada, con la subescala victimización $(\rho=.56)$, lo que indica que ambas posiciones están altamente relacionadas. Respecto a la correlación entre las variables, tanto la subescala de agresión como victimización se relacionaron de forma significativa y positiva con las dimensiones globales percepción del conflicto interparental, dificultades en la regulación emocional y con la subescala incertidumbre, así como de forma significativa y negativa con la subescala certeza, habiendo obtenido dichas variables mayores índices de correlación con la subescala victimización.

Respecto a la correlación entre subescalas, la puntuación total del instrumento de acoso escolar se relacionó de forma significativa con todas las subescalas del instrumento de percepción del conflicto interparental, siendo mayor en relación a la vivencia de culpabilidad ante el conflicto $(\rho=.25)$. En relación a la desregulación emocional, se obtuvieron índices de correlación más altos con la subescala descontrol $(\rho=.31)$, estrategias $(\rho=.29)$ e interferencia $(\rho=.28)$. En relación a la mentalización, se obtuvo un índice de correlación más alto con la subescala incertidumbre $(\rho=.27)$. La subescala victimización obtuvo correlaciones significativas con todas las subescalas del instrumento de percepción del conflicto interparental, siendo mayor en relación a la vivencia de culpabilidad ante el conflicto $(\rho=.24)$. En relación a la desregulación emocional, se obtuvieron índices de correlación más altos con las subescalas descontrol $(\rho=.29)$, interferencia $(\rho=.29)$ y rechazo emocional $(\rho=.25)$. En relación a la mentalización, se obtuvo una mayor correlación con la subescala incertidumbre $(\rho=.27)$. La subescala agresión, obtuvo correlaciones significativas con las subescalas del instrumento de percepción del conflicto interparental referentes a las propiedades del conflicto $(\rho=.15)$ y vivencia de culpabilidad $(\rho=.17)$. En relación a la desregulación emocional, se obtuvieron índices de correlación más altos con las subescalas descontrol $(\rho=.25)$, uso de estrategias $(\rho=.21)$ e interferencia emocional $(\rho=.20)$. En relación a la mentalización, se obtuvo una correlación significativa con ambas subescalas, relacionándose de forma positiva con la subescala incertidumbre $(\rho=.19)$ y de forma negativa con la escala certeza $(\rho=-.20)$.

Para el planteamiento de los modelos de mediación, dado el número elevado de variables disponibles para el modelo y de la complejidad de las relaciones entre cada uno de los indicadores, se optó por seleccionar la combinación más reducida y simple posible con el fin de respetar lo establecido por el principio de parsimonia. Así, dada las correlaciones significativas obtenidas tanto entre las subescalas como entre las puntuaciones totales, se utilizaron las puntuaciones totales del instrumento de percepción de conflicto interparental y del instrumento de dificultades en la regulación emocional. Respecto a la variable función reflexiva, se utilizó para el modelo la subescala incertidumbre, debido a la relación positiva y los valores de correlación más altos obtenidos con las variables propuestas para los modelos, a diferencia de la subescala certeza. Finalmente, se optó por utilizar la puntuación total y las subescalas relativas a la agresión y victimización del instrumento de bullying, con el fin de poder obtener modelos para cada rol, así como para para los dos roles de forma conjunta. Así, se comprobaron tres modelos de mediación encadenada formados por la puntuación total de la 
Tabla 1. Descriptivos, fiabilidad y bondad de ajuste de los instrumentos

\begin{tabular}{|c|c|c|c|c|c|c|}
\hline & $M$ & $D T$ & Rango & $\alpha$ & $\omega$ & K. S. \\
\hline EBIPQ & 6.11 & 6.51 & $0-30$ & .84 & .85 & $3.44 * *$ \\
\hline Victimización & 3.61 & 4.24 & $0-26$ & .80 & .81 & $4.15^{* *}$ \\
\hline Agresión & 2.50 & 3.42 & $0-21$ & .79 & .81 & $4.54 * *$ \\
\hline CPIC & 53.41 & 12.06 & $36-96$ & .91 & .91 & $2.35 * *$ \\
\hline Propiedades & 24.23 & 6.70 & $16-45$ & .83 & .83 & $2.93 * *$ \\
\hline Vivencia & 18.46 & 5.17 & $12-34$ & .71 & .72 & $3.01 * *$ \\
\hline Culpabilidad & 10.71 & 3.41 & $8-24$ & .83 & .84 & $4.45 * *$ \\
\hline DERS & 56.10 & 17.52 & $27-119$ & .90 & .91 & $2.08 * *$ \\
\hline Conciencia & 17.98 & 5.17 & $6-30$ & .72 & .73 & 1.09 \\
\hline Descontrol & 12.22 & 5.38 & $6-30$ & .81 & .85 & $2.84 * *$ \\
\hline Rechazo emocional & 7.88 & 3.71 & $4-20$ & .84 & .85 & $2.85 * *$ \\
\hline Interferencia & 12.78 & 4.73 & $5-25$ & .74 & .77 & $2.41 * *$ \\
\hline Claridad & 9.04 & 3.42 & $4-20$ & .70 & .71 & $1.79 * *$ \\
\hline Estrategias & 14.16 & 5.58 & $7-34$ & .75 & .78 & $2.67 * *$ \\
\hline \multicolumn{7}{|l|}{$\mathrm{RFQ}^{*}$} \\
\hline Certeza & .97 & .79 & $0-3$ & .73 & .74 & $2.21 * *$ \\
\hline Incertidumbre & .59 & .64 & $0-3$ & .71 & .76 & $3.81 * *$ \\
\hline
\end{tabular}

Tabla 2. Acoso escolar y su correlación con las variables mediadoras y la variable predictora: Coeficiente de correlación de Spearman

\begin{tabular}{|c|c|c|c|c|c|c|c|c|c|c|c|c|c|c|c|c|}
\hline & \multicolumn{3}{|l|}{ EBIPQ } & \multicolumn{4}{|c|}{ CPIC } & \multicolumn{5}{|c|}{ DERS } & \multicolumn{4}{|c|}{ RFQ } \\
\hline & 1 & 2 & 3 & 4 & 5 & 6 & 7 & 8 & 9 & 10 & 11 & 12 & 13 & 14 & 15 & 16 \\
\hline 1.EBIPQ & 1 & & & & & & & & & & & & & & & \\
\hline 2.Víctimización & $.91^{* *}$ & 1 & & & & & & & & & & & & & & \\
\hline 3.Agresión & $.81^{* *}$ & $.56^{* *}$ & 1 & & & & & & & & & & & & & \\
\hline 4.CPIC & $.19^{* *}$ & $.19^{* *}$ & $.11^{*}$ & 1 & & & & & & & & & & & & \\
\hline 5.Propiedades & $.15^{* *}$ & $.13^{* *}$ & $.15^{* *}$ & $.84 * *$ & 1 & & & & & & & & & & & \\
\hline 6.Vivencia & $.10^{* *}$ & $.14^{* *}$ & -.00 & $.81 * *$ & $.52 * *$ & 1 & & & & & & & & & & \\
\hline 7.Culpabilidad & $.25^{* *}$ & $.24 * *$ & $.17^{* *}$ & $.55^{* *}$ & $.28 * *$ & $.32 * *$ & 1 & & & & & & & & & \\
\hline 8.DERS & $.33^{* *}$ & $.34 * *$ & $.22 * *$ & $.43^{* *}$ & $.45^{* *}$ & $.46^{* *}$ & $.33^{* *}$ & 1 & & & & & & & & \\
\hline 9.Conciencia & -.01 & -.02 & -.03 & .13 & $.22 * *$ & $.10^{*}$ & .03 & .25 & 1 & & & & & & & \\
\hline 10.Descontrol & $.31 * *$ & $.29 * *$ & $.25^{* *}$ & $.37 * *$ & $.29 * *$ & $.24 * *$ & $.25^{* *}$ & $.79 * *$ & $.19^{*}$ & 1 & & & & & & \\
\hline $\begin{array}{l}\text { 11.Rechazo } \\
\text { emocional }\end{array}$ & $.20^{* *}$ & $.25^{* *}$ & .07 & $.38 * *$ & $.21 * *$ & $.32^{* *}$ & $.34 * *$ & $.63^{* *}$ & -.01 & $.38 * *$ & 1 & & & & & \\
\hline 12.Interferencia & $.28 * *$ & $.29 * *$ & $.20^{* *}$ & $.45^{* *}$ & $.32 * *$ & $.28^{* *}$ & $.25^{* *}$ & $.81^{* *}$ & $.13 * *$ & $.59 * *$ & $.42 * *$ & 1 & & & & \\
\hline 13.Claridad & $.11^{* *}$ & $.12^{* *}$ & .05 & $.33 * *$ & $.26 * *$ & $.24 * *$ & .03 & $.56^{* *}$ & $.50 * *$ & $.37 * *$ & $.24 * *$ & $.33 * *$ & 1 & & & \\
\hline 14.Estrategias & $.29 * *$ & $.31^{* *}$ & $.21^{* *}$ & $.50 * *$ & $.32 * *$ & $.31 * *$ & $.24 * *$ & $.86^{* *}$ & $.11^{*}$ & $.61 * *$ & $.51 * *$ & $.57 * *$ & $.43 * *$ & 1 & & \\
\hline 15.Certeza & $-.20 * *$ & $-.18 * *$ & $-.20 * *$ & $-.18^{* *}$ & -.08 & $-.18 * *$ & $-.12 *$ & $-.27 * *$ & -.10 & -.13 & $-.21 * *$ & -.13 & $-.18^{*}$ & -.13 & 1 & \\
\hline 16.Incertidumbre & $.27 * *$ & $.27 * *$ & $.19^{* *}$ & $.20 * *$ & $.10 *$ & $.20 * *$ & $.18 * *$ & $.39^{* *}$ & .06 & $-.29 * *$ & $.30 * *$ & $.25 * *$ & $.23^{* *}$ & $-.25^{* *}$ & $.55^{* *}$ & 1 \\
\hline
\end{tabular}

Nota. $* p<.05$. **p<.01. EBIPQ=Cuestionario del Proyecto Europeo de Intervención en Acoso Escolar; CPIC=Escala de Percepción de los hijos/as del Conflicto Interparental; DERS=Escala de dificultades en la Regulación Emocional.

escala conflicto interparental como variable predictora, las variables incertidumbre y dificultad en la regulación emocional como variables mediadoras, y la puntuación total de acoso escolar, la subescala victimización y la subescala agresión como variables resultado, respectivamente para cada uno de los tres modelos. Los resultados obtenidos para los modelos de mediación múltiple propuestos se presentan en la Tabla 3.
Únicamente el modelo 1 (variable resultado: puntuación total en acoso escolar) y el modelo 3 (variable resultado: subescala de victimización) obtuvieron efectos indirectos significativos, obteniendo correlaciones significativas entre las variables del modelo 1 ; conflicto interparental e incertidumbre $(\beta=.012)$, conflicto interparental y desregulación emocional $(\beta=.575)$, incertidumbre y desregulación emocional $(\beta=8.48)$, desregulación emocional y acoso escolar $(\beta=.102)$ 
Tabla 3. Resultados de los modelos de mediación propuestos

\begin{tabular}{|c|c|c|c|c|c|c|c|c|c|c|c|c|c|c|c|c|}
\hline & \multicolumn{2}{|c|}{ Coef. $a_{1}$} & \multicolumn{2}{|c|}{ Coef. $\mathrm{a}_{2}$} & \multicolumn{2}{|c|}{ Coef. $b_{1}$} & \multicolumn{2}{|c|}{ Coef. $b_{2}$} & \multicolumn{2}{|c|}{ Coef. d } & \multicolumn{3}{|c|}{ Efecto total } & \multicolumn{3}{|c|}{ Efecto directo } \\
\hline & $\beta$ & $p$ & $\beta$ & $p$ & $\beta$ & $p$ & $\beta$ & $p$ & $\beta$ & $p$ & $\beta$ & $S E$ & $p$ & $\beta$ & $S E$ & $p$ \\
\hline Mod. 1 & .012 & .000 & .575 & .000 & 1.244 & .018 & .102 & .000 & 8.488 & .000 & .119 & .026 & .000 & .035 & .029 & .223 \\
\hline Mod. 2 & .012 & .000 & .575 & .000 & .516 & .076 & .022 & .054 & 8.488 & .000 & .034 & .014 & .017 & .012 & .016 & .438 \\
\hline Mod. 3 & .012 & .000 & .575 & .000 & .727 & .030 & .079 & .000 & 8.488 & .000 & .085 & .017 & .000 & .022 & .018 & .221 \\
\hline
\end{tabular}

Nota. Mod. 1=Modelo 1=Conflicto Interparental (VI), Incertidumbre (VM1), Desregulación Emocional (VM2), Acoso Escolar (VD); Mod. 2=Modelo 2=Conflicto Interparental (VI), Incertidumbre (VM1), Desregulación Emocional (VM2), Agresión (VD); Mod. 3=Conflicto Interparental (VI), Incertidumbre (VM1), Desregulación Emocional (VM2), Victimización (VD).

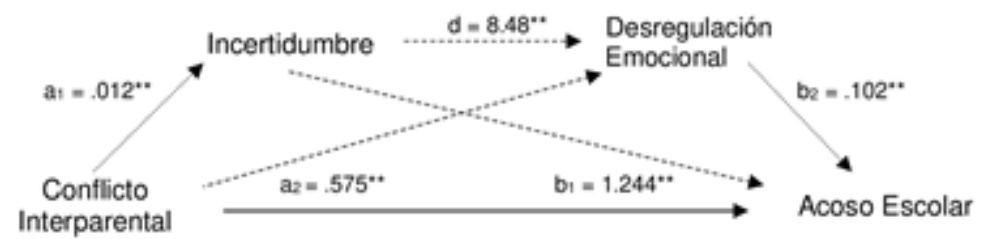

Figura 1. Modelo de Mediación de la Incertidumbre y Desregulación Emocional en la relación entre la percepción de Conflicto Interparental y el Acoso Escolar

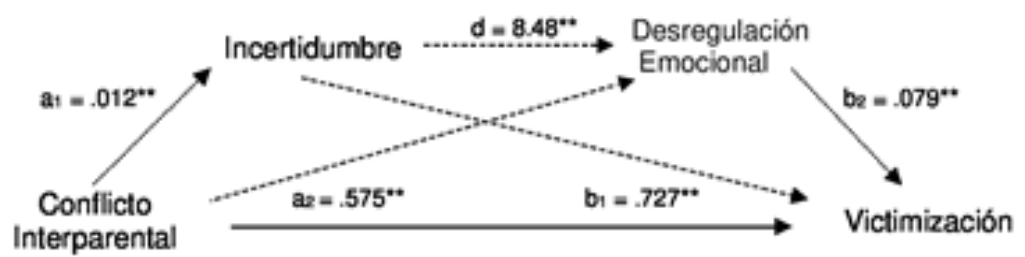

Figura 2. Modelo de Mediación de la Incertidumbre y Desregulación Emocional en la relación entre la percepción de Conflicto Interparental y Victimización

Tabla 4. Efectos indirectos de los modelos de mediación

\begin{tabular}{|c|c|c|c|c|c|c|c|c|c|}
\hline & \multicolumn{9}{|c|}{ Efecto Indirecto } \\
\hline & \multicolumn{3}{|c|}{ Ind. 1} & \multicolumn{3}{|c|}{ Ind. 2} & \multicolumn{3}{|c|}{ Ind. 3} \\
\hline & $\beta$ & $S E$ & $C I$ & $\beta$ & $S E$ & $C I$ & $\beta$ & $\overline{S E}$ & $C I$ \\
\hline Mod. 1 & .0152 & .0083 & [.0022 a .0337] & .0588 & .0155 & [.0303 a .0909] & .0106 & .0040 & [.0041 a .0197] \\
\hline Mod. 2 & .0063 & .0048 & [-.0013 a .0172] & .0131 & .0072 & {$[-.0008$ a .0278$]$} & .0024 & .0015 & {$[-.0001$ a .0056$]$} \\
\hline Mod. 3 & .0089 & .0053 & [.0002 a .0210] & .0456 & .0111 & [.0257 a .0686] & .0082 & .0031 & [.0034 a .0152] \\
\hline
\end{tabular}

y entre incertidumbre y acoso escolar $(\beta=1.224)$, así como entre las variables del modelo 3 ; entre conflicto interparental e incertidumbre $(\beta=.012)$ conflicto interparental y desregulación emocional $(\beta=.575), \quad$ incertidumbre y desregulación emocional $(\beta=8.48)$, desregulación emocional y victimización $(\beta=.079) \quad y$ entre incertidumbre $y$ victimización $(\beta=.079)$. Los resultados en ambos casos muestran un efecto de mediación total, desapareciendo el efecto directo respectivamente al introducir las variables mediadoras $(\beta=.035$; $p=.223$ y $\beta=.012 ; p=.438)$ como muestra el efecto total $(\beta=.119 ; p=.000$ y $\beta=.034 ; p=.017)$.

En la Tabla 4 se muestran los datos específicos a los efectos indirectos relativos a las variables mediadoras en cada uno de los tres modelos. En ambos modelos se obtuvieron una mayor potencia en el efecto indirecto 2 , relativo a la dificultad en regulación emocional, tanto para el modelo 1, con resultados para la comparación entre el efecto indirecto $1 \mathrm{y}$ el efecto indirecto 2 ; $\beta=-.0807$; se $=.0363$; CI [-.1515 a -.0090], entre el efecto indirecto $1 \mathrm{y}$ el efecto indirecto $3 ; \beta=.0085$; se $=.0151$; CI [-.0189 a .0408$]$, el efecto indirecto $2 \mathrm{y}$ el efecto indirecto $3 ; \beta=.0892$; se $=.0269$; $\mathrm{CI}$ [.0413 a .1460], como para el modelo 3, con resultados para la comparación entre el efecto indirecto $1 \mathrm{y}$ el efecto indirecto 2 ; $(\beta=-.0368$; se $=.0131$; CI [-.0627 a -.0118], el efecto indirecto 1 $\mathrm{y}$ el efecto indirecto $3 ; \beta=-.0007$; se $=.0053$; CI [.0099 a .0116], el efecto indirecto 2 y el efecto indirecto $3 ; \beta=.0374$; se $=.0105$; CI $[.0188$ a .0595]. En la Figura 1 se representa el modelo de mediación múltiple para el modelo 1 y en la Figura 2, el modelo de mediación encadenada para el modelo 3. 
Los resultados obtenidos respecto a los efectos indirectos muestran la significación estadística del efecto mediador de las variables incertidumbre y desregulación emocional, en la relación entre el conflicto interparental y el acoso escolar para la puntuación total así como para la victimización, mostrando en la comparación de los efectos indirectos una mayor potencia en el efecto que ejerce la regulación emocional tanto para el modelo 1 como para el modelo 3 .

\section{Discusión}

El objetivo de este estudio ha sido comprobar el rol mediador de las variables mentalización y regulación emocional en la relación entre el conflicto interparental percibido y acoso en contexto escolar, contemplando la victimización y agresión de forma separada y de forma conjunta. Los resultados obtenidos han puesto de manifiesto el papel mediador de las variables propuestas en el acoso escolar contemplando de forma conjunta victimización y agresión, así como victimización de forma separada, no así en el caso de la agresión de forma separada al no haberse obtenido un efecto mediador significativo en este modelo. Los resultados obtenidos se discuten a continuación a la luz de la investigación existente, teniendo en cuenta las implicaciones para la investigación futura y la práctica clínica.

En primer lugar, los resultados obtenidos ponen de manifiesto la relación entre las variables estudiadas, mostrando una relación entre la percepción de conflicto parental con el acoso escolar, siendo más significativa con la puntuación total en el instrumento de acoso escolar y con la subescala victimización, y esto a su vez con una mayor desregulación emocional en los menores, así como dificultad en la habilidad de mentalización, tendiente a una incertidumbre en los estados mentales propios y de los otros. Estos resultados son consistentes con la literatura, la cual respalda la relación entre las variables propuestas, entre conflicto interparental y acoso escolar contemplado los roles de victimización y agresión (Martínez et al., 2009; Mistli, 2017), conflicto interparental y regulación emocional (Gong, 2013), regulación emocional y acoso escolar contemplado ambos roles (Estévez et al., 2018) así como mentalización en relación a regulación emocional y victimización en el acoso escolar (Morosan et al., 2020), y de forma indirecta entre mentalización y conductas de agresión (Morosan et al., 2020) y acoso escolar (Mahady-Wilton et al., 2000; Twemlow \& Fonagy, 2006). Es escasa la literatura que pone de manifiesto la relación entre el conflicto interparental y la habilidad de mentalización en los menores, siendo un mayor foco de estudio en la relación de conflicto parental y la capacidad de mentalización parental (Jessee et al., 2018) por la influencia de esta variable en la relación de padremadre e hijo/a (Gordo, Iriarte, \& MartínezPampliega, 2020).

En esta línea, las relaciones entre las variables obtenidas permitieron analizar la hipótesis central del estudio, referente al efecto mediador de las variables regulación emocional y mentalización, concretamente la incertidumbre de los estados mentales o hipomentalización en el desarrollo de conductas de acoso (en su conjunto, para agresión y para victimización) ante la percepción de conflicto interparental en adolescentes. Los resultados obtenidos muestran apoyo a la hipótesis del estudio de forma parcial, habiendo obtenido resultados significativos en relación al acoso escolar contemplado en su totalidad y la victimización en el acoso escolar, no habiendo obtenido resultados significativos ante las conductas de agresión en acoso escolar. Los resultados indican por tanto, que ante la mayor percepción de conflicto interparental por parte de los adolescentes, contemplando dentro de esta percepción las propiedades del conflicto, la vivencia y la culpabilidad sentida, estos serán más propensos a presentar dificultades en la capacidad de regulación emocional y mentalización, mostrando en relación a esta última, una tendencia a la incertidumbre de los estados mentales propios y ajenos o hipomentalización. Estas dificultades en ambas habilidades presentadas en contexto de conflicto interparental explican, según el modelo obtenido, que los menores se vean envueltos en situación de acoso escolar, contemplado esta variable desde el continuo de este constructo así como específicamente en el rol de víctima.

Estos resultados se entienden a la luz de la literatura existente. A pesar de que son escasos los estudios que señalan la relación entre conflicto interparental y la capacidad de mentalización en 
los hijos/as, tanto la mentalización como la habilidad para regular las emociones se desarrollan en el contexto de seguridad en la relación de apego, principalmente derivado del entendimiento y desarrollo de los estados emocionales del niño por su cuidador (Marszał y Jańczak, 2017), seguridad que puede verse mermada en situación de conflicto interparental. En este contexto, debido al conflicto, la disponibilidad parental se ve reducida, mostrando mayores dificultades en la capacidad de observar las necesidades, representar y mantener los estados internos de los/as menores e influyendo en la capacidad de desarrollo de ambas habilidades (Fonagy \& Target, 1997; Gong, 2013; Marszał \& Jańczak, 2017). De acuerdo con la hipótesis de seguridad emocional, los menores desarrollarían estrategias poco adaptativas de regulación emocional como respuesta ante su necesidad de aumentar su seguridad emocional mermada debido a la presencia de conflicto interparental (Gong, 2013; Warmuth et al., 2018), influenciado por la incertidumbre de los estados mentales, ya que una correcta capacidad de mentalización, ofrece una representación simbólica de los estados afectivos que permite lidiar con las emociones difíciles a nivel intrapsíquico, en vez de hacerlo a través de formas desadaptativas e impulsivas de actuar estas emociones (Marszał \& Jańczak, 2017). Así, la dificultad en ambas habilidades, se relaciona con el acoso escolar entendido como un continuo, contemplado los roles de agresión, victimización y ambos de forma conjunta (Salmivalli, 2010), así como de forma separada victimización. En relación a la emisión de conductas agresivas, la dificultad en ambas capacidades se relaciona con una dificultad a la hora de inhibir los comportamientos violentos (Bateman et al., 2013; Fonagy \& Luyten, 2009), fruto de las difíciles emociones experimentadas en casa y del aprendizaje de utilizar estrategias poco adaptativas. Respecto a las víctimas, a pesar de que la literatura existente que permite entender la relación entre la victimización en contexto escolar y la dificultad en la capacidad de mentalización y regulación emocional es escasa, sí se señala la relación de ambas dificultades con la capacidad para desarrollar estrategias de enfrentamiento efectivas y respuestas emocionales apropiadas y flexibles, aumentando el riesgo de rechazo por parte de sus compañeros (Estévez et al., 2018; Mahady-Wilton et al., 2000). En esta línea, los menores podrían elegir enmascarar sus emociones ante la exposición de conflicto interparental con el fin evitar implicación y hostilidad (Shelton \& Harold, 2008), tratando de mantener su seguridad emocional.

En conclusión, los resultados obtenidos en el presente estudio han permitido poner de manifiesto la relación directa entre las variables propuestas, subrayando la complejidad del acoso escolar, entendido como un continuo desde la agresión y la victimización más que como entidades separadas (Salmivalli, 2010; Unnever, 2005), permitiendo esclarecer la relación entre el desarrollo del acoso escolar, especialmente en la posición de víctima, ante el conflicto interparental, así como entender la importancia del periodo adolescente para el desarrollo de estrategias adaptativas como factores protectores ante dificultades como el acoso escolar. Otro de los puntos importantes, ha sido la inclusión de la variable mentalización contemplado dos de las dificultades con las que puede manifestarse, hipomentalización e hipermentalización, permitiendo entender mejor el constructo.

Una mejor comprensión en la relación entre las variables permite favorecer el incremento de la responsabilidad de educadores y profesionales, en colaboración con la implicación de los padres, en el proceso de desarrollo y mantenimiento de las conductas de acoso, facilitando el desarrollo de intervenciones más adaptadas a las necesidades de los adolescentes. También señalar la importancia del desarrollo programas psicoeducativos relativos a la comprensión de los estados mentales propios y de los demás y, en especial, al desarrollo de recursos dirigidos a utilizar estrategias adecuadas de control de las emociones y de respuestas en situaciones vividas como intensas emocionalmente, así como intervenciones dirigidas al trabajo con padres que doten de recursos con los que poder lidiar con situaciones de conflicto de una forma más constructiva, promoviendo espacios seguros donde seguir conteniendo las necesidades de los menores.

Con todo ello y de cara a futuros estudios, se considera relevante seguir profundizando en la relación entre las variables, permitiendo esclarecer los mecanismos que tienen lugar entre ellas, 
especialmente dentro del acoso escolar así como en situación de victimización. Por otra parte, se propone el estudio de este fenómeno contemplando la agresión-victimización como rol combinado de forma más específica, así como el estudio del cyberbullying, con el fin de comprobar si los resultados de este estudio son generalizables en esta variante del acoso escolar. Finalmente, y dada la importancia de la capacidad de mentalización parental en el desarrollo de esta capacidad en los menores, sería interesante poder contemplar esta variable en futuros estudios.

No obstante, este estudio debe ser interpretado teniendo en cuenta ciertas limitaciones. La principal limitación está vinculada al instrumento utilizado para medir el acoso escolar, teniendo en cuenta su carácter cuantitativo, sin conceder una categorización más exacta de los distintos roles y sin permitir esclarecer las diferencias entre ellos salvo en relación a las conductas de acoso. Esta limitación pone de manifiesto la complejidad del acoso escolar. Otra de las limitaciones es el carácter transversal del estudio, siendo interesante la ampliación de la muestra procedente de otros centros escolares. Del mismo modo, sería interesante plantear estudios longitudinales que permitiesen indagar de una forma más profunda en la influencia del conflicto interparental en las variables propuestas. En este sentido, sería importante considerar en otros estudios la presencia de más informantes como pueden ser padres y profesores con el fin de recabar una mayor información de los procesos propuestos. Finalmente, se señala como otra de las limitaciones el no ser un estudio multi-método, habiendo utilizado el autoinforme como único método a la hora de recabar información, lo que supone que los resultados pueden estar influidos por la deseabilidad social, tal y como hacen referencia otros estudios (Garaigordobil \& Aliri, 2013).

\section{Referencias}

Ahmed, E., \& Braithwaite, V. (2004). Bullying and victimization: Cause of concern for both families and schools. Social Psychology of Education, 7, 35-54. doi:10.1023/B:SPOE.0000010668.43236.60

Allen, J. P. (2008). The attachment system in adolescence. In J. Cassidy, P. R. Shaver, J.
Cassidy, y P. R. Shaver (Eds.), Handbook of attachment: Theory, research, and clinical applications (2nd ed., pp. 419-435). New York: Guilford Press.

Bateman, A., Bolton, R., \& Fonagy, P. (2013). Antisocial personality disorder: A mentalizing framework. Focus - American Psychiatric Publishing, 11, 178-186.

doi:10.1176/appi.focus.11.2.178

Bateman, A., \& Fonagy, P. (2012). Introduction and overview. En Fonagy, P., Bateman, A. y Luyten, P. (Eds.), Handbook of mentalizing in mental health practice (pp. 3-42). Arlington, VA: American Psychiatric Publishing.

Benbassat, N., \& Priel, B. (2015) Why is fathers' reflective function important? Psychoanalytic Psychology, 32(1), 1-22.

doi:10.1037/a0038022

Blakemore, S. J. (2008). The social brain in adolescence. Nature Reviews. Neuroscience, 9(4), 267-277. doi:10.1038/nrn2353.

Bleiberg, E. (2013). Mentalizing-based treatment with adolescents and families. Child and Adolescent Psychiatric Clinics of North America, 22, 295-330. doi:10.1016/j.chc.2013.01.001.

Brighi, A., Ortega, R., Scheitauer, H., Smith, P. K., Tsormpatzoudis, C., Barkoukis, V., Del Rey, R., et al. (2012). European Bullying Intervention Project Questionnaire (EBIPQ). University of Bologna. Unpublished Manuscript.

Buelga, S., Iranzo, B., Cava, M. J., \& Torralba, E. (2015). Psychological profile of adolescent cyberbullying aggressors. International Journal of Social Psychology, 30(2), 382-406. doi:dx.doi.org/10.1080/21711976.2015.1016754

Christie-Mizell, C. (2003) Bullying: The consequences of interparental discord and child's self-concept. Family process, 42(2), 237-251.

doi:doi.org/10.1111/j.1545-

5300.2003.42204.x

Cohen, J. (1988). Statistical power analysis for the behavioral sciencies. Hillsdale, NJ: Erlbaum.

Cummings, E. M., \& Davies, P. T. (2010). Marital conflict and children: An emotional security perspective. New York, NY: The Guilford Press. 
Cummings, E. M., George, M. R. W., McCoy, K. P., \& Davies, P. T. (2012). Interparental conflict in kindergarten and adolescent adjustment: Prospective investigation of emotional security as an explanatory mechanism. Child Development, 83(5), 17031715 . doi:10.1111/j.1467-8624.2012.01807.x.

Davies, P. T., \& Cummings, E. M. (1994). Marital conflict and child adjustment: An emotional security hypothesis. Psychological Bulletin, 116, 387-411. doi:10.1037/0033-2909.116.3.387

Davies, P. T., \& Martin, M. J. (2013). The reformulation of emotional security theory: The role of children's social defense in developmental psychopathology. Development and Psychopathology, 25, 14351454. doi:dx.doi.org/10.1017/S0954579413000709

Delgado, A., Romero, L., Antolín-Suárez, L., \& Parra, Á. (2016). Conflictos interparentales, estilos parentales y problemas interiorizados en hijos e hijas adolescentes. Revista Mexicana de Psicología, 33(2), 101-110. Recuperado de http://www.redalyc.org/pdf/2430/2430560440 02.pdf

Due, P., Holstein, B. E., Lynch, J., Diderichsen, F., Nic Gabhein, S., Scheidt, P., Currie, C., \& The Health Behaviour in School-Aged Children Bullying Working Group. (2005). Bullying and symptoms among school-aged children: International comparative cross sectional study in 28 countries. The European Journal of Public Health, 15, 128-132. doi:10.1093/eurpub/cki105

Espelage, D. L., \& Swearer, S. M. (2003). Research on school bullying and victimization: What have we learned and where do we go from here? School Psychology Review, 32(3), 365-383.

Estévez, C., Carrillo, A., \& Gómez, M. D. (2018). Inteligencia emocional y bullying en escolares de primaria. International Journal of Developmental and Educational Psychology, 1(1), 227-238. doi:10.17060/ijodaep.2018.n1.v1.1200

Fonagy, P. (1991). Thinking about thinking: Some clinical and theoretical considerations in the treatment of a borderline patient. International Journal of Psychoanalysis, 72, 639-656.

Fonagy, P., \& Luyten, P. (2009). A developmental, mentalization-based approach to the understanding and treatment of borderline personality disorder. Development and Psychopathology, 21, 1355-1381. doi:10.1017/S0954579409990198

Fonagy, P., Luyten, P., Moulton-Perkins, A., Lee, Y., Warren, F., Howard, S., \& Lowyck, B. (2016). Development and validation of a selfreport measure of mentalizing: The reflective functioning questionnaire. PLOS ONE, 11(7), 28. doi:10.1371/journal.pone.0158678

Fonagy, P., Steele, H., \& Steele, M. (1991). Maternal representations of attachment during pregnancy predict the organization of infantmother attachment at one year of age. Child Development, 62, 891-905. http://dx.doi.org/10.2307/1131141

Fonagy, P., \& Target, M. (1997). Attachment and reflective function: Their role in selforganization. Development and Psychopatology, 9(04), 679-700.

Garaigordobil, M., \& Aliri, J. (2013). Cibracoso ("cyberbullying") en el País Vasco: diferencias de sexo en víctimas, agresores y observadores. Behavioral Psychology/ Psicologia Conductual, 21, 461-474.

Goeke-Morey, M., Cummings, E., \& Papp, L. (2007). Children and marital conflict resolution: Implications for emotional security and adjustment. Journal of Family Psychology, 21, 744-753.

Gómez-Simón, I., Penelo, E., \& de la Osa, N. (2014). Factor structure and measurement invariance of the difficulties emotion regulation scale (DERS) in Spanish adolescents. Psicothema, 26(3), 401-408. doi:10.7334/psicothema2013.324

Gong, X. (2013). Relations among interparental conflict, parenting practices, and emotion regulation during emerging adulthood (tesis doctoral). Ball State University, Muncie.

Gordo, L., Iriarte, L., \& Martínez-Pampliega, A. (2020) Versión española del Cuestionario de Función Reflexiva Parental (CFRP-18). Revista Iberoamericana de Diagnóstico y Evaluación - e Avaliação Psicológica, 55(2), 5-17. doi:doi.org/10.21865/RIDEP54.1.01 
Gratz, K. L., \& Roemer, L. (2004). Multidimensional assesment of emotion regulation and dysregulation: Development, factor structure and initial validation of the dificulties in emotion regulation scale. Journal of Psychopathology and Begavioral Assesment, 6(1), 41-54. doi:10.1023/B:JOBA.0000007455.08539.94

Grych, J. H., Seid, M., \& Fincham, F. D. (1992). Assessing marital conflict from the child's perspective: The children's perception of interparental conflict scale. Child Development, 63, 558-572. doi: $10.2307 / 1131346$

Harold, G. T., Shelton, K. H., Goeke-Morey, M., \& Cummings, E. M. (2004). Marital conflict, child emotional security about family relationships and child adjustment. Social Development, 13(3), 350-376. doi:10.1111/j.1467-9507.2004.00272.x

Hayes, A. F., \& Preacher, K. J. (2013). Conditional process modeling: Using structural equation modeling to examine contingent causal processes. In G. R. Hancock \& R. O. Mueller (Eds.) Structural equation modeling: A second course (2nd $\mathrm{Ed}$ ). Greenwich, CT: Information Age Publishing.

Holt, M., Finkelhor, D., \& Kaufman, G. (2007) Hidden forms of victimization in elementary students involved in bullying. School Psychology Review, 36(3), 345-360.

Ingoldsby, E., Shaw, D. S., \& García, M. (2001). Intrafamily conflict in relation to boys's adjustment at school. Development and Psychopatology, 13(1), 35-12. doi:10.1017/S0954579401001031

Iraurgi, I., Martínez-Pampliega, A., Sanz, M., Cosgaya, L., Galíndez, E., \& Muñoz, A. (2006). Escala de conflicto interparental desde la perspectiva de los hijos (CPICS): Adaptación al español y estudio de validación de una versión abreviada de 36 ítems. Letras de Deusto, 115(37), 149-168.

Iraurgi, I., Martínez-Pampliega, A., Sanz, M., Cosgaya, L., Galíndez, E., \& Muñoz, A. (2008). Escala de conflicto interparental desde la perspectiva de los hijos (CPIC): Estudio de una versión abreviada de 36 ítems. Revista Iberoamericana de Diagnóstico y Evaluación - e Avaliação Psicológica, 25(1), 9-34.
Recuperado

https://www.aidep.org/sites/default/files/201707/R251.pdf

Jessee, A., Mangelsdorf, S. C., Wong, M. S., Schoppe-Sullivan, S., Shigeto, A., \& Brown, G. L. (2018). The role of reflective functioning in predicting marital and coparenting quality. Journal of Child and Family Studies, 27(1), 187-197. doi:dx.doi.org/10.1007/s10826-017-0874-6

Keenan, K. (2000). Emotion dysregulation as a risk factor for child psychopathology. Clinical Psychology: Science and Practice, 7(4), 418434. doi:10.1093/clipsy.7.4.418

Kelly, B. M., Schwartz, D., Gorman, A. H., \& Nakamoto, J. (2008). Violent victimization in the community and children's subsequent peer rejection: The mediating role of emotion dysregulation. Journal of Abnormal Child Psychology, 36(2), 175-185. doi:10.1007/s10802-007-9168-6

Kochenderfer-Ladd, B., \& Skinner, E. A. (2002). Children's coping strategies: Moderators of the effects of peer victimization? Developmental Psychology, 38(2), 267-278. doi:10.1037//0012-1649.38.2.267

Koss, K. J., George, M. R. W., Bergman, K. N., Cummings, E. M., Davies, P. T., \& Cicchetti, D. (2011). Understanding children's emotional processes and behavioral strategies in the context of marital conflict. Journal of Experimental Child Psychology, 109(3), 336352. doi:10.1016/j.jecp.2011.02.007

Krishnakumar, A., \& Buehler, C. (2000). Interparental conflict and parenting behaviors: A meta-analytic review. Family Relations, 49(1), 25-44.

doi:10.1111/j.1741-3729.2000.00025.x

Lyons-Ruth, K., Bronfman, L., \& Atwood, G. (1999). A relational diathesis model of hostile helpless states of mind: Expressions in mother-infant interaction. In J. Solomon \& C. C. George (Eds.), Attachment disorganization (pp. 33-70). New York, NY: Guilford Press.

Mistli, G. L. (2017). Influencia del clima escolar y familiar en el acoso escolar y cibernético de universitarios. Revista Mexicana de Investigación en Psicología, 9(1), 31-44.

Mahady-Wilton, M. M., Craig, W. M., \& Pepler, D. J. (2000). Emotional regulation and display 
in classroom victims of bullying: Characteristic expressions of affect, coping styles and relevant contextual factors. Social Development, 9(2), 226-245. doi:10.1111/1467-9507.00121

Marszał, M., \& Jańczak, A. (2017). Emotion dysregulation, mentalization and romantic attachment in the nonclinical adolescent female sample. Current Psychology: A Journal for Diverse Perspectives on Diverse Psychological Issues, 37(4), 894-904. doi:10.1007/s12144-017-9573-0

Martínez, B., Musitu, G., Amador, L., \& Murgui, S. (2009). Conflicto marital, comunicación familiar y ajuste escolar en adolescentes. Revista Mexicana de Psicología, 26(1), 27-40.

Morris, A. S. (2000). The role of the family context in the development of children's emotion regulation (tesis doctoral). Temple Univeristy, Philadelphia.

Morris, K. L. (2007). Familiar antecedents of bullying and victimization: The moderating role of social support (tesis doctoral). University of California: California.

Morosan, L., Fonseca-Pedrero, E., \& Debbané, M. (2020). Network analysis of reflective functioning and conduct problems during adolescence. Psychology of Violence, 10(3), 300-311. doi:10.1037/vio0000258

Ortega-Ruíz, R., Del Rey, R., \& Casas, J.A. (2016). Evaluar el bullying y el ciberbullying validación española del EBIP-Q y del ECIPQ. Psicología Educativa, 22(1), 71-79. doi:10.1016/j.pse.2016.01.004

Pope, A. W., \& Bierman, K. L. (1999). Predicting adolescent peer problems and antisocial activities: The relative roles of aggression and dysregulation. Developmental Psychology, 35(2), 335-346. doi:10.1037/0012-1649.35.2.335

Salmivalli, C. (2010). Bullying and the peer group: A review. Aggression and Violent Behavior, 15(2), 112-120. doi:10.1016/j.avb.2009.08.007

Save the Children (Ed.). (2016). Yo a eso no juego. Recuperado de https://www.savetheChildren.es/publicaciones /yo-eso-no-juego-bullying-y-ciberbullying-enla-infancia
Save the Children. (Ed.). (2013). Acoso escolar y ciberacoso: Propuesta para la acción. Recuperado de https://www.savetheChildren.es/sites/default/f iles/imce/docs/acoso_escolar_y_ciberacoso_in forme_vok_-_05.14.pdf

Schwartz, D., Petit, G., Dodge, K., \& Bates, J. (2000). Friendship as a moderating factor in the pathway between early harsh home environment and later victimization in the peer group. Developmental Psychology, 36(5), 646-662. doi:10.1037/0012-1649.36.5.646

Shelton, K., \& Harold, G. (2008). Pathways between interparental conflict and adolescent psychological adjustment: Bridging links through children's cognitive appraisals and coping strategies. The Journal of Early Adolescence, 28(4), 555-582. doi:10.1177/0272431608317610

Thompson, R. (1994). Emotion regulation: A theme in search of definition. In N. Fox (Ed.), The development of emotion regulation: Behavioral and biological considerations. Monographs of the Society for Research in Child Development, 59, 25-52.

Trizano-Hermosilla, I., \& Alvarado, J. M. (2016). Best alternatives to Cronbach's Alpha reliability in realistic conditions: Congeneric and asymmetrical measurements. Frontiers in Psychology, 7. https://doi.org/10.3389/fpsyg.2016.00769

Tur-Porcar, A., Mestre, V., Samper, P., \& Malonda, E. (2012). Crianza y agresividad de los menores: ¿Es diferente la influencia del padre y de la madre? Psicothema, 24(2), 284288.

Twemlow, S. W., \& Fonagy, P. (2006). Transforming violent social systems into nonviolent mentalizing systems: An experiment in schools. In J. G. Allen \& P. Fonagy (Eds.), The handbook of mentalization-based treatment (p. 289-306). John Wiley \& Sons Inc. doi: doi.org/10.1002/9780470712986.ch15

UNESCO (2018) School violence and bullying: global status and trend, drivers and consequences. Recuperado de infocoponline.es/pdf/BULLYING.pdf

Unnever, J. D. (2005): Bullies, aggressive victims and victims: Are they distinct groups? 
Aggressive behaviour, 31(2), pp. 153-171. doi:10.1002/ab.20083

Warmuth, K. A., Cummings, E. M., \& Davies, P. T. (2018). Child behavioral dysregulation as a mediator between destructive marital conflict and children's symptoms of psychopathology. Journal of Child and Family Studies. doi:10.1007/s10826-018-1038-z

Werneck, H., Eder, M. O., Yanagida, T., \& Rollett, B. (2014). Predicting adolescents' parent-child relationship quality from parental personality, marital conflict and adolescents' personality. European Journal of Developmental Psychology, 11(2), 159-176. doi:10.1080/17405629.2013.876914. 